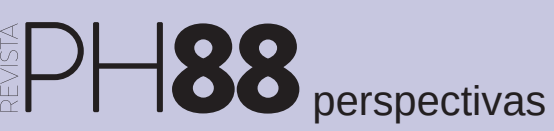

a debate La eficiencia energética y la edificación histórica | coordinan Mónica López Sánchez, Ana Yáñez Vega

\title{
Eficiencia energética y edificación histórica. Hacia la ciudad europea del futuro desde la ciudad histórica
}

\author{
Ana Yáñez, directora de la Fundación de Casas Históricas y Singulares \\ URL de la contribución <www.iaph.es/revistaph/index.php/revistaph/article/view/3673>
}

Mónica López Sánchez, coordinadora del Área de Industrias Culturales y Patrimonio de la Fundación Ars Civilis

La búsqueda de reducción de costes de mantenimiento y la mejora del confort son necesidades básicas de los residentes en edificios y áreas urbanas históricas. Ello no constituye ninguna novedad ya que siempre ha sido una pretensión de las personas, residan en edificios históricos o no. ¿Cómo afrontar estas aspiraciones en la actualidad para prevenir la pérdida de residentes en inmuebles históricos?

En estos momentos, la eficiencia energética, en todos los sentidos y también en el ámbito de la edificación, es una de las principales prioridades en Europa y, por tanto, también en España. Esto implica que se movilicen cuantiosos recursos económicos para estos fines y que se fomente el desarrollo de actuaciones que redunden en ellos, de manera destacada en los núcleos urbanos. Se persigue intervenir sobre el parque edificado en muchas ciudades y localidades más pequeñas que también cuentan con áreas urbanas e inmuebles protegidos. ¿Cómo está siendo abordada la aspiración de reducción de consumos energéticos, las soluciones planteadas para ello y las intervenciones concretas cuando existen bienes culturales protegidos? Esta es la otra cuestión esencial que nos ha llevado a proponer el debate de la sección Perspectivas de este número de revista ph y este texto.

Pretendemos plantear una visión global que facilite la aproximación a los diferentes ámbitos desde los que se puede tratar este tema y que permita al lector profundizar en aquellos que más le interesen a través de los especialistas en los mismos ${ }^{1}$. Cuáles son las políticas existentes en la materia y las normativas para desarrollarlas, qué fuentes y mecanismos de financiación las soportan, qué conocimiento técnico y soluciones se están desarrollando, cómo pueden plantearse estrategias de actua- ción a nivel local para la ejecución de las actuaciones y cómo gestionarlas, cuáles son los retos en formación, educación y difusión... Estas son las preguntas que nos hemos hecho como punto de partida para la reflexión que incluimos a continuación.

Hablaremos de ideas y acciones. Las ideas se reflejan en estrategias políticas $y$, en el caso de la eficiencia energética, van a venir planteadas en primera instancia desde la Unión Europea. No vamos a abordar la historia reciente y los condicionantes que han desembocado en el punto en que nos encontramos; nos referiremos exclusivamente al marco actual y al caso español.

La estrategia Europa $2020^{2}$ es el paraguas que está guiando toda la acción de la Unión Europea en estos momentos y lo hará durante los próximos años. En ella la reducción de emisiones de efecto invernadero y el aumento de la eficacia energética son prioridades básicas (CE, 2013: 3); en ambos casos el objetivo quedó fijado en alcanzar un 20\%, aunque estos datos han sufrido revisiones a posteriori (PLAN, 2014: 3). Todas las políticas europeas están canalizadas para contribuir a alcanzar los objetivos de la Europa 2020, de ahí el protagonismo que las cuestiones asociadas a la eficiencia energética y a la economía baja en carbono tienen en estos momentos.

La aportación nacional al cumplimiento de los objetivos de Europa 2020 va a venir recogida cada año en el Programa Nacional de Reformas de España ${ }^{3}$, un instrumento muy útil para entender toda la política nacional. No obstante, la "herramienta central de la política energética española" es el Plan Nacional de Acción de Eficiencia Energética 2014-2020 (PLAN, 2014: 4). 
Una vez determinadas las ideas, la visión, estas son canalizadas por medio de la normativa, que permitirá concretar las reglas del juego, acotar las acciones y marcar las vías y los instrumentos disponibles para desarrollar las actuaciones que permitan alcanzar el objetivo fijado para que la visión que se persigue se convierta en una realidad. En el caso de la eficiencia energética, la normativa que va a fijar unos mínimos en estrategias y requisitos técnicos es de ámbito europeo por lo que se materializa a través de directivas, figura de obligado cumplimiento para los Estados de la Unión y que establece unos mínimos comunes para todos los Estados, que podrán decidir tanto los medios que van a emplear para cumplirlos como ir más allá de esos mínimos. El Plan Nacional de Acción de Eficiencia Energética 2014-2020 nace para cubrir uno de los requisitos de la Directiva 2012/27/UE del Parlamento y del Consejo, de 25 de octubre de 2012, relativa a la eficiencia energética. La normativa europea sobre eficiencia energética excluye de la obligatoriedad de su cumplimiento a los edificios protegidos por su interés histórico, dando prioridad al mantenimiento de los valores que los hacen merecedores de dicha protección y dejando en manos de los Estados la posibilidad de exigirlos o no.

Por otro lado, la situación del patrimonio cultural es muy distinta. Entendido como integrante de la cultura, es un ámbito exclusivo de los países, donde la Unión Europea únicamente puede realizar una política de acompañamiento, de apoyo, coordinación y complemento de las políticas nacionales de cada Estado miembro. Por tanto, no va a haber legislación europea de obligado cumplimiento en materia directa de patrimonio cultural (salvo para la exportación de bienes culturales y la restitución de los que hayan salido de manera ilegal) pero sí determinadas orientaciones, instrumentos y medidas incentivadoras importantes sobre todo en el ámbito de la investigación.

Como decíamos, las directivas son de obligado cumplimiento para los Estados miembro por lo que han de ser traspuestas a la legislación nacional. Para conocer la estructura nacional que traspone la normativa europea y las especificidades propias para el caso de España, recomendamos la lectura de la contribución de la profesora de la Universidad de Málaga, Isabel González, al debate de este número con el título "Algunas consideraciones sobre el marco normativo de la eficiencia energética en los edificios históricos" y las referencias bibliográficas que acompaña. Como recoge González, tenemos una legislación de patrimonio cultural que no menciona las cuestiones energéticas, una legislación europea de eficiencia energética que excluye a la edificación protegida por su interés histórico, pero una legislación nacional que no lo hace expresamente y que plantea lagunas o debilidades.

El posicionamiento de la eficiencia energética como una de las prioridades políticas esenciales de la Unión Europea y, por ende, de los Estados miembro supone una cuantiosa asignación de recursos económicos destinados a cumplir o, al menos, aproximarse lo máximo posible a los objetivos fijados en relación con la misma.

La financiación va a ir dirigida fundamentalmente a proyectos de investigación, desarrollo e innovación orientada al mercado, por un lado, y a la realización de actuaciones concretas, sobre todo por parte de las administraciones públicas, por otro. Con el apoyo a los proyectos se persigue aumentar el conocimiento y que este se traslade a nuevos productos y servicios que lleguen al mercado, el cual será promovido y apoyado mediante la dotación de financiación para actuaciones concretas. Se trata en definitiva de destinar recursos económicos que conduzcan al crecimiento y consolidación del mercado asociado a la eficiencia energética, al tiempo que a lograr que los hábitats urbanos sean más sostenibles. Los retos en este ámbito son varios y entre ellos no podemos olvidar las dificultades de los usuarios finales de estos nuevos productos y servicios tanto para adquirirlos como incluso para conocer su existencia.

En el caso de España, la dedicación de recursos a la eficiencia energética ${ }^{4}$ en la edificación va unida además a otra prioridad, la reconversión y recuperación del sector de la construcción, como viene recogido en la Ley 
8/2013, de 26 de junio, de Rehabilitación, Regeneración y Renovación Urbanas. Esta ley es el marco del que surgen los tres programas del Ministerio de Fomento dirigidos al fomento de la rehabilitación edificatoria, la regeneración y renovación urbanas y al fomento de ciudades sostenibles y competitivas. Estos programas están orientados en buena medida a las intervenciones para la mejora de la eficiencia energética en los inmuebles ${ }^{5}$. Destacamos esta ley porque es el marco que sienta las bases para las operaciones de carácter urbano, por tanto, de mayor escala que un inmueble individualmente considerado, bajo la figura de las Áreas de Regeneración y Renovación Urbanas (ARRUs). Pero también porque a pesar de la fecha de su aprobación, relativamente reciente, apenas si se hace referencia en ella a los bienes protegidos por su interés histórico, sobre los que las comunidades autónomas tienen las competencias más importantes.

Otro paquete de recursos económicos destinado a la eficiencia energética en la edificación es gestionado por el Instituto para la Diversificación y Ahorro de la Energía (IDAE), que depende del Ministerio de Industria, Energía y Turismo. Este organismo canaliza la mayor parte de los recursos existentes en España para actuaciones de eficiencia energética, gestionando el Fondo Nacional de Eficiencia Energética (168 millones de euros) y el Fondo JESSICA-F.I.D.A.E. (cerca de 123 millones de euros) ${ }^{6}$. EI primero consta de cuatro líneas de actuación: alumbrado exterior municipal, PYMEs y grandes empresas del sector industrial, transporte y rehabilitación energética de edificios. Esta última línea es el denominado Programa PAREER, en funcionamiento desde 2013 pero que a partir de 2015 deja de estar limitado a usos de vivienda u hotelero y pasa a denominarse PAREER-CRECE, y que contempla además la posibilidad de sumar dotación económica vía fondos FEDER en una serie de casos concretos, entre los que se encuentra que las actuaciones se realicen sobre edificios incluidos en ARRUs.

El IDAE es el organismo intermedio que tiene encomendada la gestión de los fondos FEDER (siglas del Fondo Europeo de Desarrollo Regional) destinados a eficien- cia energética. A este marco pertenece el fondo de cartera F.I.D.A.E. (Fondo de Inversión en Diversificación y Ahorro de Energía), creado en 2011 entre el IDAE y el Banco Europeo de Inversiones para financiar proyectos que persigan un desarrollo urbano sostenible y la mejora de la eficiencia energética y uso de energías renovables. Para poder utilizar los fondos estructurales para inversiones reembolsables se ha recurrido al instrumento financiero JESSICA, creado para áreas urbanas.

En las diferentes vías de financiación existentes hasta el momento no se contempla la especificidad de los inmuebles históricos protegidos, cuyo estatus conlleva una serie de limitaciones a la hora de actuar sobre los mismos que dificultan o hacen inviable acceder a estas ayudas, además de encarecer considerablemente las intervenciones. Llevamos ya varios años llamando la atención del IDAE sobre la necesidad de dedicar recursos para los inmuebles y conjuntos protegidos; desde la entidad se han reconocido esas dificultades y la necesidad de abordar la situación pero no se terminan de materializar medidas concretas. Lo cual nos lleva a continuar con estos esfuerzos y a animar a todos los agentes implicados a contribuir a dar visibilidad a esta cuestión. No es lo mismo rehabilitar energéticamente un inmueble o acometer actuaciones eficientes desde un punto de vista energético si dicho edificio se encuentra protegido por sus valores culturales, que hay que preservar de acuerdo con la normativa sobre patrimonio cultural, que si no lo está. Esta situación debería ser asumida por aquellos operadores que intervienen en la financiación y tienen capacidad de decisión sobre los requisitos y circunstancias que se tienen en cuenta para optar y adjudicar la financiación.

Hemos mencionado ya los fondos FEDER, uno de los instrumentos creados por la Unión Europea para el desarrollo de su política de cohesión territorial. En el actual período de programación, 2014-2020, un volumen muy destacado de los fondos para España tienen una orientación hacia la energía. Aún no están aprobados todos los programas operativos regionales ni nacionales; es sin duda una oportunidad para la dotación de recursos hacia el tema que nos ocupa si conseguimos llamar la aten- 


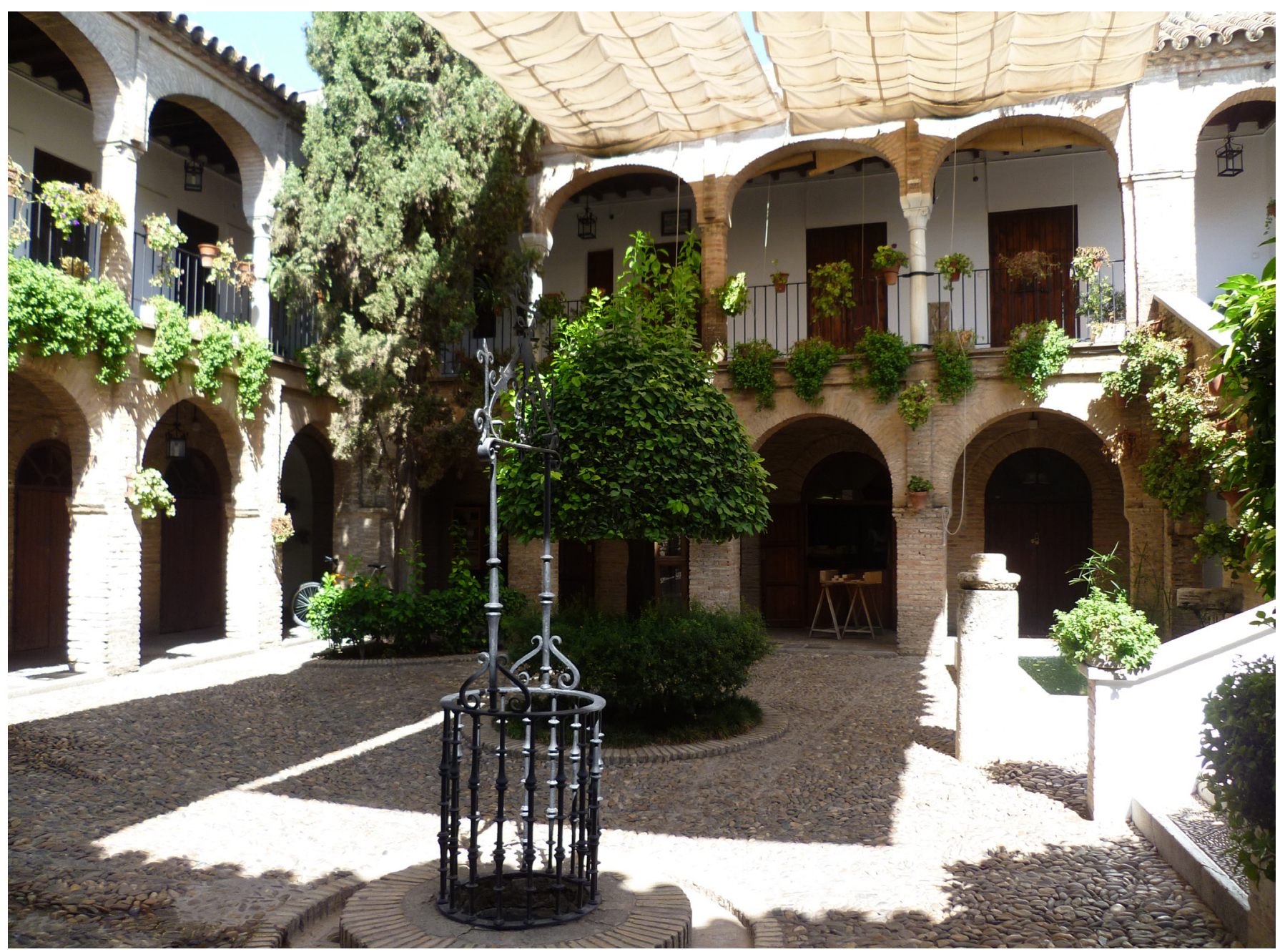

Históricamente el ser humano ha buscado soluciones para mejorar el confort ante las condiciones climáticas; en la imagen la tipología de casa en torno a un patio central que se complementa con vegetación, agua y sombra para reducir los efectos del calor, solución típicamente mediterránea | foto Mónica López Sánchez, 2010

ción sobre ello y demostrar la necesidad y el interés para las administraciones de apoyar y acometer actuaciones que supongan un avance en este sentido. El mensaje es muy claro: ahora es el momento, puesto que el ciclo de inversiones está comenzando.

Llegados a este punto cabría preguntarnos si se sabe cómo intervenir en los inmuebles protegidos, con la certeza irrenunciable de que se haga preservando el carácter, los valores y la integridad histórica de ese patrimonio cultural.
El principal escollo en este sentido es que los procedimientos y recursos empleados en la actualidad para los consumos y usos energéticos de los inmuebles están planteados para la edificación coetánea y de nueva construcción, por lo que no son válidos para la histórica. Y ello porque estos procedimientos pueden poner en riesgo los valores culturales de los edificios históricos, no siendo autorizados en ocasiones por los departamentos de cultura de las diferentes comunidades autónomas. A esta circunstancia se suma el desconocimiento sobre la dinámica interna de los edificios históricos y el uso cotidiano 


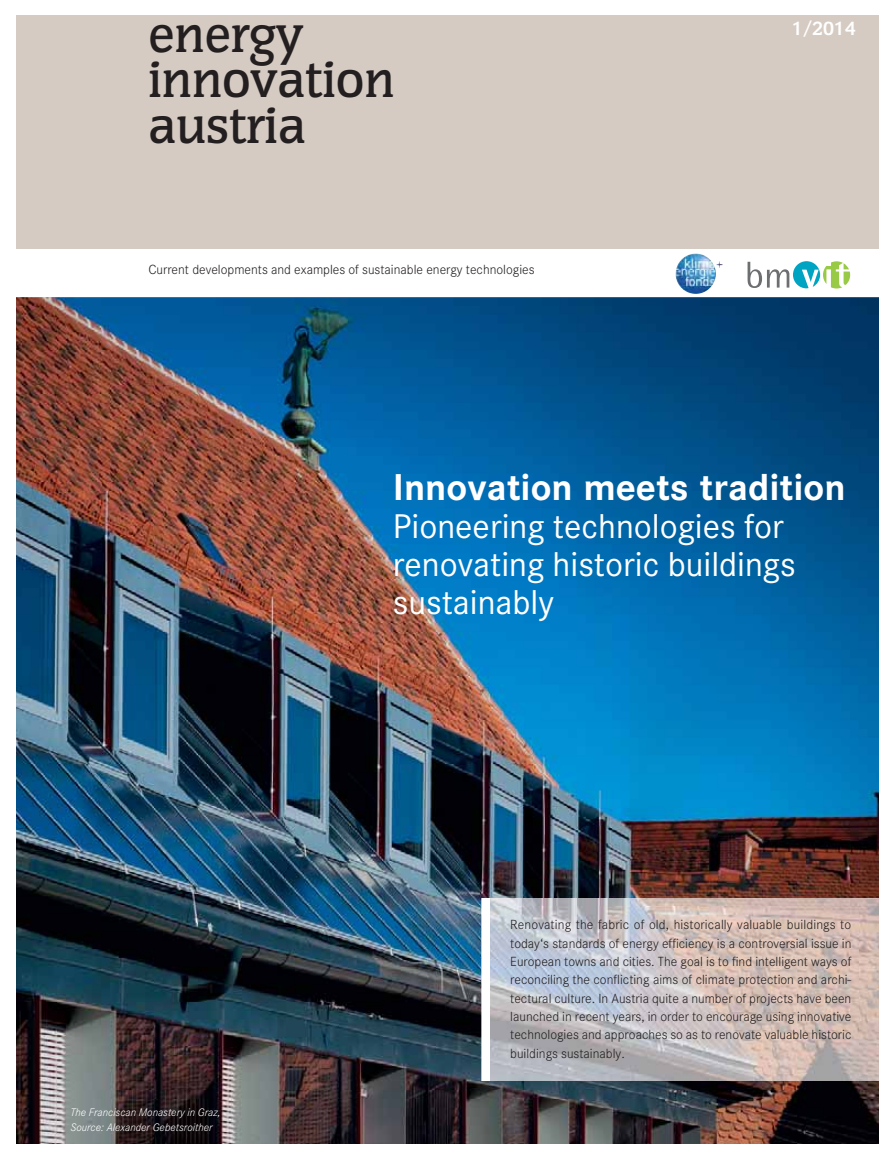

Austria es uno de los países donde la Administración central no ha eludido la problemática de la mejora de la eficiencia energética en la edificación histórica. Documento disponible en <https://www.klimafonds.gv.at/assets/Uploads/ Broschren/Energy-Innovation-Austria/Innovation-meets-tradition-EIA-1-2014.pdf> [Consulta: 28/09/2015]

de los habitantes para quienes fueron construidos desde el punto de vista energético, así como la existencia de múltiples tipologías arquitectónicas adaptadas a diferentes territorios en función del clima y de los materiales disponibles para su construcción en cada momento.

La edificación histórica presenta una complejidad a la que es ajena la arquitectura de nueva construcción por lo que el mercado se ha centrado en proveer soluciones para esta última, dejando de lado aquella que requería mayores inversiones previas en investigación y formación de personal. Ha sido gracias a la atención prestada por profesionales fundamentalmente procedentes

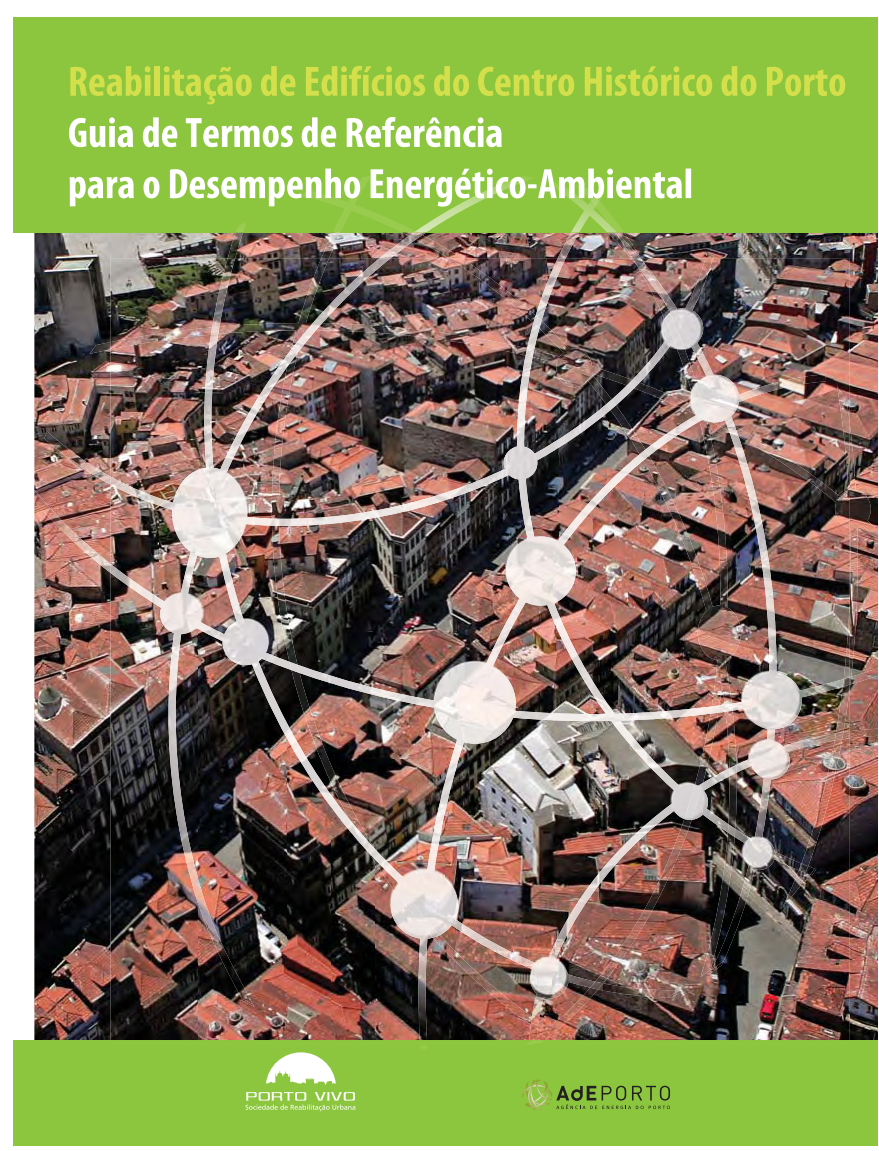

Porto es un ejemplo paradigmático de una estrategia de recuperación de un centro histórico que incorpora las cuestiones energéticas y las afronta en un marco de colaboración entre los agentes implicados y con competencias tanto en eficiencia energética como en patrimonio cultural | foto portada de la Guia de Termos de Referência para o Desempenho Energético-Ambiental (2010)

de la arquitectura y la ingeniería y dedicados a la investigación, que se está avanzando en los últimos años en conocimiento y en soluciones ajustadas a las especificidades de la edificación protegida. Además, en este ámbito han de convivir nuevos recursos provenientes de las últimas investigaciones con fórmulas tradicionales basadas en conocer el funcionamiento de este tipo de inmuebles: unir ambas formas de conocimiento no es fácil y requiere en ocasiones de la presencia de equipos multidisciplinares para intervenir de manera correcta.

Podemos considerar que la situación en este sentido es positiva, ya que el número de profesionales que están 
_a debate La eficiencia energética y la edificación histórica | coordinan Mónica López Sánchez, Ana Yáñez Vega

dedicando esfuerzos a aumentar el conocimiento relacionado con energía y edificación histórica sigue una progresión ascendente. Además, cada vez son más los equipos que trabajan con tipologías arquitectónicas de las que mayor presencia hay en la Península Ibérica y condiciones ambientales también propias de estas zonas.

No obstante, no es sólo cómo intervenir sino también, y antes que ello, en función del qué y el para qué. En este sentido, recogemos palabras expresadas por el grupo de investigación dirigido por José Luis González que en otro número de esta revista planteaban lo siguiente: "Hay que diferenciar la motivación inicial del planteamiento energético por parte de los promotores, en función de si se pretende únicamente acreditar el estado energético del edificio a efectos de una transacción inmobiliaria, o bien si se requiere un aumento del confort ambiental, una reducción del gasto, del consumo, o de la demanda, una mejora de la relación entre el confort y el gasto, o combinaciones de estas posibilidades. También hay que considerar si la motivación inicial del planteamiento energético de los bienes culturales inmuebles se debe a un cambio de uso del edificio, o bien se pretende mantener el uso existente. A su vez, cada uno de estos aspectos requiere enfoques específicos por parte de los distintos agentes intervinientes en los edificios históricos, refiriéndonos fundamentalmente a los gestores o promotores, a los técnicos, o bien a los usuarios de la arquitectura." (GONZÁLEZ MORENO-NAVARRO; DOTOR NAVARRO; MORROS CARDONA et ál., 2013: 21). Como se ha puesto de manifiesto, el uso tanto original como actual y futuro del edificio histórico es fundamental para intervenir con rigor y adecuadamente desde la perspectiva de la eficiencia energética. Además, son necesarios esos enfoques específicos, que requieren de profesionales formados en diversas áreas de conocimiento. En este sentido, sería muy oportuno que los planes de estudios de diferentes ámbitos (arquitectura, ingeniería, ciencias sociales, humanidades) incorporaran nociones sobre el comportamiento energético de los edificios históricos y sobre la importancia de conjugar la eficiencia energética con los valores culturales de dichos inmuebles, así como sobre la formulación de políticas públicas que tuvieran en cuenta ambos aspectos.

Otra de las cuestiones fundamentales para que este tema tenga un largo recorrido es el de una educación y una difusión adaptadas a los diferentes tipos de usuarios de la información y de las soluciones que se propongan. La eficiencia energética ya es por sí mismo un tema complejo que requiere de buenas dosis de imaginación y rigor para ser divulgado de manera amena. Si a ello le añadimos las dificultades para comprender los diferentes grados de protección de los bienes históricos, así como los derechos y obligaciones que ello conlleva, el resultado es un cóctel difícil de digerir si no se realiza un gran esfuerzo para que sea comprendido por quienes tienen que hacer uso de estas nuevas soluciones a las que nos hemos referido. Habrá que aprovechar conceptos cercanos, como el de ciudad o el de casa, para construir discursos que pongan de relieve la importancia de casar las necesidades de eficiencia energética con las de proteger los inmuebles que incorporan valores culturales.

Uno de los retos más importantes en estos momentos tiene que ver con la gestión, con cómo implementar todas las actuaciones e incluso cómo gastar los presupuestos comprometidos; y esta es una cuestión que va más allá de la edificación histórica para afectar a las áreas urbanas en su conjunto.

Están definidos cuáles son los objetivos, los recursos económicos y las soluciones técnicas, pero no existe tanta claridad sobre qué estrategias definir y cómo ejecutarlas, cómo incorporar a todos los agentes implicados para hacerles partícipes y protagonistas del proceso, y de manera destacada a los residentes y propietarios de los inmuebles.

En el caso de la edificación histórica, son abundantes las actuaciones en edificios con un grado de protección que permite acometer actuaciones básicas como las renovaciones de ventanas o de calderas (a partir de los denominados Planes Renove, promovidos por el IDAE con los organismos regionales competentes en materia 
energética que son quienes los gestionan en sus respectivos territorios) y existen casos puntuales en los que se han ensayado actuaciones más integrales, casi siempre en inmuebles declarados bienes de interés cultural de grandes dimensiones y frecuentemente no de uso residencial. Sin embargo, las decisiones en materia energética transcurren en paralelo a las de preservación del patrimonio histórico, rara vez fusionándose y aún menos frecuente siendo incorporadas como una política decidida dentro de los planes de conservación y gestión del patrimonio cultural. No podemos dejar de mencionar, en este sentido, la experiencia de la ciudad portuguesa de Oporto, en cuyo plan de manejo de la ciudad protegida ha sido incorporada una estrategia de mejora de la eficiencia energética en los inmuebles históricos que ha demostrado que es posible que los responsables políticos y técnicos de las decisiones en materia de eficiencia energética y en materia de patrimonio cultural, respectivamente, trabajen conjuntamente para definir criterios comunes y simplificar y facilitar los procedimientos de cara a los usuarios finales.

Concluimos planteando la necesidad de ahondar en un debate amplio que conduzca a la inclusión de las cuestiones energéticas en las políticas de patrimonio cultural y en los planes de gestión de los bienes que pudieran verse afectados, especialmente en el caso de las áreas urbanas protegidas. Cabe esperar que si se consigue introducir la eficiencia energética como una de las líneas de trabajo en los planes de gestión de los conjuntos históricos, los resultados puedan tener mayor alcance: en el catálogo de soluciones a medida para cada caso, en número de inmuebles adecuadamente intervenidos, en capacidad de captación de recursos económicos para financiar las actuaciones $y$, en definitiva, en calidad de vida y confort para los habitantes de los edificios y barrios históricos.

\section{NOTAS}

1. Una aproximación al estado de la cuestión reciente sobre la temática y a los profesionales que están trabajando en ella desde diferentes enfoques puede encontrarse en los participantes y las contribuciones presentadas al Congreso Internacional de Eficiencia Energética y Edificación Histórica (celebrado en Madrid durante los días 29-30 de septiembre de 2014). En las direcciones <http://www.energy-heritage. com> y <http://energyheritage.wordpress.com> están disponibles el programa del congreso, las actas y los vídeos autorizados de las ponencias. Para 2016 se prevé la realización de la segunda edición del congreso.

2. La web oficial de la Estrategia Europa 2020 es <http://ec.europa. eu/europe2020/index_es.htm>

3. Los documentos que recogen esos programas nacionales están disponibles en el archivo digital de la Presidencia del Gobierno <http:// www.lamoncloa.gob.es/>

4. La relación de las medidas de apoyo de la Administración General del Estado a la eficiencia energética puede encontrarse en el Plan Nacional de Acción de Eficiencia Energética 2014-2020 (PLAN, 2014: 153-154).

5. Los detalles de estas ayudas pueden conocerse, dentro de la web oficial del Ministerio de Fomento <http://www.fomento.es> en la sección de la Dirección General de Arquitectura, Vivienda y Suelo, que es el departamento de quien depende el plan de ayudas a la rehabilitación de edificios; o en la web habilitada para dicho plan <http://www. fomento.gob.es/rehabilitacion>

6. Información ampliada disponible en la web oficial del IDAE <http:// www.idae.es/>. Las cifras mencionadas han sido extraídas de esa página web. 


\section{BIBLIOGRAFÍA}

- COMISIÓN EUROPEA (CE) (2010) Comunicación de la Comisión - EUROPA 2020 Una estrategia para un crecimiento inteligente, sostenible e integrador. Bruselas, 3.3.2010; COM (2010) 2020 final

- COMISIÓN EUROPEA(CE) (2013) Comprender las políticas de la Unión Europea Europa 2020: la estrategia europea de crecimiento. Luxemburgo: Oficina de Publicaciones de la Unión Europea, 2013

- DIRECTIVA 2012/27/UE del Parlamento y del Consejo, de 25 de octubre de 2012, relativa a la eficiencia energética, por la que se modifican las Directivas 009/125/CE y 2010/30/UE, y por la que se derogan las Directivas 2004/8/CE y 2006/32/ CE. Diario Oficial de la Unión Europea, de 14 de noviembre de 2012, [L 315/1]

- GONZÁlEZ MORENO-NAVARRO, J. L.; DOTOR NAVARRO, A.; MORROS CARDONA, J.; et ál. (2013) El difícil equilibrio entre eficiencia energética y conservación de los valores patrimoniales en edificios históricos. Revista ph [en línea], n. ${ }^{\circ}$ 84, 2013 pp. 20-21, <www.iaph.es/revistaph/index. php/revistaph/article/view/3393> [Consulta: 12/08/2015]

- Plan Nacional de Acción de Eficiencia Energética 20142020 (2014) [en línea] Versión de 30 de abril de 2014. Ministerio de Industria, Energía y Turismo (MINETUR)-Secretaría de Estado de Energía, 2014 <https://ec.europa.eu/energy/sites/ ener/files/documents/NEEAP_2014_ES-es.pdf> [Consulta: 05/06/2015]

- LÓPEZ, M.; YÁÑEZ, A.; GOMES DA COSTA, S. et ál. (coord.) (2014) Actas del Congreso Internacional de Eficiencia Energética y Edificación Histórica / Proceedings of the International Conference on Energy Efficiency and Historic Buildings (Madrid, 29-30 Sep. 2014). Madrid: Fundación de Casas Históricas y Singulares y Fundación Ars Civilis, 2014 